\title{
EFFICIENT GENERATION OF MAXIMAL IDEALS IN POLYNOMIAL RINGS
}

\author{
BY \\ E. D. DAVIS $\left({ }^{1}\right)$ AND A. V. GERAMITA $\left({ }^{2}\right)$
}

\begin{abstract}
The cardinality of a minimal basis of an ideal $I$ is denoted $\nu(I)$. Let $A$ be a polynomial ring in $n>0$ variables with coefficients in a noetherian (commutative with $1 \neq 0$ ) ring $R$, and let $M$ be a maximal ideal of $A$. In general $\nu\left(M A_{M}\right)+1>\nu(M)>\nu\left(M A_{M}\right)$. This paper is concerned with the attaining of equality with the lower bound. It is shown that equality is attained in each of the following cases: (1) $A_{M}$ is not regular (valid even if $A$ is not a polynomial ring), (2) $M \cap R$ is maximal in $R$ and (3) $n>1$. Equality may fail for $n=1$, even for $R$ of dimension 1 (but not regular), and it is an open question whether equality holds for $R$ regular of dimension $>1$. In case $n=1$ and $\operatorname{dim}(R)=2$ the attaining of equality is related to questions in the $K$-theory of projective modules. Corollary to (1) and (2) is the confirmation, for the case of maximal ideals, of one of the EisenbudEvans conjectures; namely, $\nu(M)<\max \left\{\nu\left(M A_{M}\right), \operatorname{dim}(A)\right\}$. Corollary to (3) is that for $R$ regular and $n>1$, every maximal ideal of $A$ is generated by a regular sequence-a result well known (for all $n>1$ ) if $R$ is a field (and somewhat less well known for $R$ a Dedekind domain).
\end{abstract}

1. Background and statement of principal results. We consider noetherian rings (commutative with $1 \neq 0$ ), denoting by $\nu(I)$ the cardinality of a minimal basis of the ideal $I$. Several papers have taken up the question of estimating $\nu$ in terms of local data. Forster's theorem [F] implies: for $M$ a maximal ideal of $A, \nu(M) \leqslant \max \left\{\nu\left(M A_{M}\right), \operatorname{dim}(A)+1\right\}$. Eisenbud and Evans [E-E] conjecture a sharpening of the Forster result for polynomial rings; their conjecture implies that in the inequality above one can replace $" \operatorname{dim}(A)+1$ " by " $\operatorname{dim}(A)$ ". We verify this case of their conjecture as a consequence of Theorem 2 (below) and the following theorem which is valid for arbitrary noetherian $A$ (proof in $\$ 2$ ).

THEOREM 1. $\nu(M)=\nu\left(M A_{M}\right)$ if $A_{M}$ is not regular. (In general $\nu\left(M A_{M}\right)<$ $\left.\nu(M) \leqslant \nu\left(M A_{M}\right)+1.\right)$

Received by the editors February 11, 1976.

AMS (MOS) subject classifications (1970). Primary 13B25; Secondary 13D15, $13 \mathrm{H} 10$.

Key words and phrases. Polynomial ring, maximal ideal, number of generators, Hilbert rings, regular sequences, projective modules, $K_{0}$, seminormal ring.

(') The first named author wishes to express his thanks to the second named author and to the Mathematics Department of Queen's University for hospitality extended on many occasions.

${ }^{2}$ ) Supported in part by the National Research Council of Canada under grant number A-8488.

- American Mathematical Society 1977 
It is well known that the lower bound is not always attained. The most classical examples occur in nonfactorial Dedekind domains. It is also well known that the lower bound is always attained in many cases wherein $A$ is a polynomial ring, e.g., when the coefficient ring is a field. We investigate the possibility of attaining the lower bound in polynomial rings and discover two more quite general sufficient conditions for the occurrence of this event (Theorems 2 and 3). We discuss the cases left open in $\$ 4$ and consider possible generalizations for rings which are not noetherian in $\$ 5$.

Henceforth, in the absence of any statement to the contrary, the following notation is in force: $A$ denotes a polynomial ring in $n>0$ variables with coefficients in a noetherian ring $R$. $M$ denotes a maximal ideal of $A$, and $P=M \cap R$.

THEOREM 2. $\nu(M)=\nu\left(M A_{M}\right)$ if $P$ is maximal. (Hence this conclusion always holds if $R$ is a Hilbert ring.)

Corollary. $\nu(M) \leqslant \max \left\{\nu\left(M A_{M}\right), \operatorname{dim}(A)\right\}$.

We prove Theorem 2 in $\$ 2$. To prove the corollary it suffices, in view of Theorem 1, to consider the case $\nu\left(M A_{M}\right)=h t(M)=\operatorname{dim}(A)$. But in this case $P$ must also be maximal, and Theorem 2 applies.

Our third, and most general, result was to us most startling:

THEOREM 3. $\nu(M)=\nu\left(M A_{M}\right)$ if $n>1$.

COROLlaRY. If $n>1$, then

(i) $\nu(M)=\operatorname{ht}(M)$ if $A_{M}$ is regular (equivalently, if $R_{p}$ is regular) and, consequently, $M$ is generated by a regular sequence in this case.

(ii) $M$ is generated by a regular sequence if $R$ is regular.

This theorem is proved in $\$ 3$. The corollary follows immediately from the theorem since a prime ideal $I$ is generated by a regular sequence if and only if $\nu(I)=\mathrm{ht}(I)[\mathrm{D}-1]$.

We shall see in $\S 4$ that (i) (and hence Theorem 3) may fail for $n=1$ and $R$ of dimension 1 , but not regular. We know of no case where (ii) fails for $n=1$.

For $R$ regular of dimension 1, in which case we may as well assume $R$ is a Dedekind domain, (ii) was proved valid for all $n$ by Endo [E] in case $R$ is semilocal, and by several mathematicians (see [D-G]) for the remaining case in which $R$ is necessarily a Hilbert ring (cf. Theorem 2 above).

When $R$ is regular semilocal of dimension 1, in which case $R$ is factorial, the statement $\nu(M)=\nu\left(M A_{M}\right)$ is valid for trivial reasons if either $P \neq(0)$ or if $P=(0)$ and $n=1$; all the work in [E] goes into the case $n>1$ and $P=(0)$. Observe that by (i) of the corollary the statement " $\nu(M)=\nu\left(M A_{M}\right)$ " is valid 
in this case without any regularity assumption on $R$. The hypothesis " $P=$ $(0)$ " forces $R$ to be a semilocal domain of dimension 1 . In $\$ 5$ we shall see that if one assumes that about $R$, then even the noetherian condition may be nontrivially weakened.

\section{Proofs of Theorems 1 and 2.}

Proof of Theorem 1. The inequality in parentheses is well known (e.g., [D-G]). To prove the first assertion of the theorem we proceed by induction on $\operatorname{ht}(M)$. Suppose $\operatorname{ht}(M)=0$. Let $I$ be the primary component of 0 having radical $M$, and let $J$ be the intersection of the remaining primary components of 0 . Since $I$ and $J$ are clearly comaximal, we have that $A$ is isomorphic to $(A / I) \oplus(A / J)$, with $M$ corresponding to the maximal ideal $(M / I) \oplus$ $(A / J)$. Hence $\nu(M)=\nu((M / I) \oplus(A / J))$. Furthermore, since $M / I \neq 0$ (because $A_{M}$ is not regular), $\nu((M / I) \oplus(A / J))=\nu(M / I)$. Observe that $A / I$ is isomorphic to $A_{M}$. Hence $\nu(M)=\nu(M / I)=\nu\left(M A_{M}\right)$. Assume now that $\operatorname{ht}(M)>0$. We show that there is an $x \in M$ such that $\operatorname{ht}(M / x A)=$ $\mathrm{ht}(M)-1$ and such that $x$ is part of a minimal basis of $M A_{M}$. Let $y \in M$ be part of such a minimal basis. Select $z \in M$ lying in every minimal prime ideal of $A$ not containing $y$, but not in any minimal prime which contains $y$. Then $x=y+z^{2}$ is as desired. Let $a \mapsto \bar{a}$ denote the canonical homomorphism $A \rightarrow A / x A$. Then $\nu\left(\overline{M A}_{\bar{M}}\right)=\nu\left(M A_{M}\right)-1$ and $\operatorname{ht}(\bar{M})=\mathrm{ht}(M)-1$; whence $\bar{A}_{\bar{M}}$ is not regular. By the induction hypothesis, $\nu(\bar{M})=\nu\left(\overline{M A}_{\bar{M}}\right)$. Clearly, $\nu(M)-1 \leqslant \nu(\bar{M})$. It follows that $\nu(M) \leqslant \nu\left(M A_{M}\right)$, and so that $\nu(M)=$ $\nu\left(M A_{M}\right)$.

In order to prove Theorem 2 we need

LEMMA 1. $\nu\left(M A_{M}\right)=\nu\left(P R_{P}\right)+n$.

Proọ. There is no loss in assuming that $R$ is local and $P$ is its maximal ideal. Then since $A / P A$ is a polynomial ring of $n$ variables with coefficients in the field $R / P$, the maximal ideal $M / P A$ is generated by a regular sequence of $n$ elements. It follows that $M A_{M} / P A_{M}$ is so generated. Now [D-2] applies to show that $\nu\left(M A_{M}\right)=\nu\left(P A_{M}\right)+n$. It is well known that $\nu(P)=$ $\nu\left(P A_{P A}\right)$ (e.g., $\left.[\mathrm{N}, 18.9]\right)$, and it is clear that $\nu(P) \geqslant \nu\left(P A_{M}\right) \geqslant \nu\left(P A_{P A}\right)$. The desired equality then follows.

Proof of Theorem 2. We proved in [D-G] that $\nu(M) \leqslant \nu\left(P R_{p}\right)+n$ if $P$ is maximal. That fact and Lemma 1 give $\nu(M)=\nu\left(M A_{M}\right)$.

REMARK. The result from [D-G] just quoted is valid even if $R$ is not noetherian, provided that $P$ is finitely generated. The same is true of the result from [D-2] used in the proof of Lemma 1. We shall need these facts for $\S 5$.

3. Proof of Theorem 3. It suffices to consider the case of $n=2$, in which case the theorem is an immediate consequence of Theorem 2 and: 
Lemma 2. Assume $n=2$ and $A=R[X, Y]$. Then $M \cap R\left[X-Y^{s}\right]$ is maximal for all sufficiently large $s$.

Proof. We consider $A=(R[Y])[X]$ and the elements of $A$ as polynomials in $X$ with coefficients in $R[Y]$. With this in mind define $L$ to be the ideal of $R[Y]$ consisting of the leading coefficients of the polynomials in $M$. Clearly $L \supseteq M \cap R[Y]=Q$, and since $M$ is not the extension of its contraction to $R[Y]$ we have that $L \neq Q$.

We claim that $L$ contains a monic polynomial. To see this observe first that $Q \neq P R[Y]$ since $\mathrm{ht}(P)+2=\mathrm{ht}(M)=\mathrm{ht}(Q)+1$. It follows that every prime ideal of $R[Y]$ properly containing $Q$ lies over a prime ideal of $R$ properly containing $P$. Hence if $P$ is maximal, then so is $Q$-in which case $L=R[Y]$ and the claim is obvious. So assume that neither $P$ nor $Q$ is maximal. Then $\operatorname{dim}(R / P)=\operatorname{dim}(R[Y] / Q)=1$ (see e.g. [A-T]). Thus, the only prime ideals, $N$, of $R[Y]$ which contain $L$ are maximal ideals of $R[Y]$ which lie over maximal ideals of $R$. Note that $R[Y] /(N \cap R) R[Y]$ is then a polynomial ring in one variable over a field, whence each of these maximal ideals must contain a monic polynomial of $R[Y]$. Thus, there is a monic polynomial in $\operatorname{rad}(L)$ and, hence, a monic polynomial in $L$.

Call this monic polynomial $f$ and let $g \in M$ be a polynomial having $f$ as its leading coefficient, i.e.,

$$
g=f_{0}+f_{1} X+\cdots+f_{m-1} X^{m-1}+f X^{m}
$$

$\left(f_{j}, f \in R[Y]\right)$.

Choose $s>\operatorname{deg} f_{j}, 0 \leqslant j \leqslant m-1$, and let $Z=X-Y^{s}$ (i.e., $X=Z+$ $\left.Y^{s}\right)$. Then $A=R[Z, Y]$ and, regarding elements of $A$ as polynomials in $Y$ with coefficients in $R[Z]$, we see that $g$ is monic in $Y . A / g A$ is then an integral extension of $R[Z]$, whence $M \cap R[Z]$ is maximal because it lies under the maximal ideal $M / g A$.

REMARK. This change of variables occurs both in [E] and in Suslin's work on the Serre problem [B], [Sw]. Our treatment permits a certain nonnoetherian extension (see $\$ 5$ ) which raises the question of to what extent Lemma 2 is a theorem in universal commutative algebra-"high school" algebra as it were.

4. Remarks on the case of $n=1$. In view of Theorems 1,2 and 3, the problem of determining whether $\nu(M)=\nu\left(M A_{M}\right)$ is open only if $P$ is nonmaximal, $A_{M}$ (equivalently, $R_{P}$ ) is regular and $n=1$. In this section we assuine that $n=1$. If $\operatorname{dim}(R)=0$, then $P$ is necessarily maximal and Theorem 2 applies. If $\operatorname{dim}(R)>2$, we know nothing beyond Theorems 1 and 2-even if $R$ is a 3-dimensional regular local domain. For regular local $R$ one readily verifies that $\nu(M)=\nu\left(M A_{M}\right)$ whenever $R / P$ is regular; and we suspect that the conclusion is valid even when $R / P$ is singular. However, if 
this proves not to be the case, then we suspect the counterexample to come with $R$ the power series ring in three variables over a field and $P$ one of Moh's bad examples [Mo].

For the rest of this section we assume that $R$ is a domain. We give a definitive answer to the open question for $\operatorname{dim}(R)=1$, and explore some interesting relationships of this question with questions in the $K$-theory of projective modules for $\operatorname{dim}(R)=2$.

Suppose $\operatorname{dim}(R)=1$. Let $J$ and $\bar{J}$ denote the Jacobson radicals of $R$ and its normalization, respectively.

THEOREM 4 (CF. [E, 3.1]). $\nu(M)=\nu\left(M A_{M}\right)$ for all $M$ if, and only if, $J=\bar{J}$.

The proof is omitted because we prove a more general theorem in $\S 5$. For semilocal $R$, the condition " $J=\bar{J}$ " is equivalent to what is now called "seminormality" (and to "all projective $A$-modules are free" [E], [B-M], [T]). The local ring at a singular point of a plane curve is seminormal if, and only if, the singularity is an ordinary double point. (See [S], also [Bo] and [D-3].) Thus, for example, if $R$ is the local ring at the origin on the curve $Y^{2}=X^{3}$, there are maximal ideals $M$ of $A$ such that $2=\nu(M)>\nu\left(M A_{M}\right)=1$.

For the rest of this section $\operatorname{dim}(R)=2$. In this case the only $M$ that need concern us are those for which $A_{M}$ is a regular local domain of dimension 2. An argument of Serre [Sr] now comes into play. Let $E=\operatorname{Ext}_{A}^{1}(M, A)$. Observe that $M A_{M}$ is generated by a regular sequence of two elements and that $M A_{Q}=A_{Q}$ for every prime ideal $Q$ of $A$ other than $M$. Thus, $\operatorname{hd}(M)=1$ and one readily verifies then-say by direct computation-that $E \otimes A_{M}=A_{M}$ and $E \otimes A_{Q}=0$. Thus, $M$ is the radical of the annihilator $I$ of $E$; and it follows that $E$ is cyclic $A$-module because it is a cyclic $(A / I)$-module. Serre's lemma then says that $M$ has a projective resolution of the form $0 \rightarrow A \rightarrow L \rightarrow$ $M \rightarrow 0$. Moreover, $\wedge^{2} L \approx A$; this is because the Koszul complex $0 \rightarrow \wedge^{2} L$ $\rightarrow L \rightarrow M \rightarrow 0$ is locally exact and, hence, exact (cf. [Fd]). Thus, since $L$ is of rank 2 , any condition forcing $L$ to be free implies that $\nu(M)=2$. This will be so in each of the cases of the following theorem.

THEOREM 5. $\nu(M)=\nu\left(M A_{M}\right)$ for all $M$ in each of the following cases:

(i) $R$ is semilocal and regular (cf. [G]).

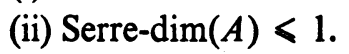

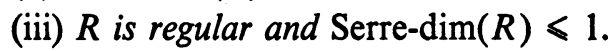

(iv) $R$ is semilocal, $2 R=R$ and $K_{0}(A) \approx \mathbf{Z} \oplus \operatorname{Pic}(A)$.

Proof of Theorem 5. In case (i) all $A$-projectives are free [M]. Recall that "Serre-dim $(S) \leqslant d$ " means that every $S$-projective of rank $>d$ has a free summand. In case (ii) then, $L \approx I \oplus A$. Then $A \approx \wedge^{2} L \approx I$; whence $L$ is free. By $[\mathbf{M}]$ and $[\mathbf{Q}]$, every $A$-projective is the extension of an $R$-projective if 
$R$ is regular and of dimension $\leqslant 2$. Hence in case (iii) Serre-dim $(A) \leqslant 1$, and (ii) applies. In case (iv) $L \oplus A^{s} \approx I \oplus A^{s+1}$. Taking $s+2$ exterior powers shows that $I \approx A$; i.e., $L$ is stably free. For the rest of the argument we adopt the notation of Swan's exposition of the Suslin-Vaserstein results [Sw, §6]. Since $\bigwedge^{2} L \approx A, L$ supports a symplectic structure. Furthermore, since $L$ is stably free of $\operatorname{rank} 2,[L]-\left[A^{2}\right] \in W(A)=\operatorname{ker}\left(K S p_{0}(A) \rightarrow K_{0}(A)\right)$. Now $W(A)=W(R)$ (Karoubi's theorem [Sw, 7.1]), and $W(R)=0$ because $R$ is semilocal. So $[L]=\left[A^{2}\right]$ in $K S p_{0}(A)$; whence $L \approx A^{2}[\mathrm{Sw}, 6.4]$.

REMARKS. (1) Since a regular ring is a direct sum of domains, (i) and (iii) of Theorem 5 remain valid even if $R$ is not a domain. (ii) and (iv) are, in general, false for nondomains, but are valid for a nondomain provided that $\operatorname{dim}(R / Q)=2$ for every minimal prime $Q$.

(2) An unpublished argument of Murthy gives the following: Let $R$ be semilocal with normalization a finite $R$-module. If $\nu(M)=\nu\left(M A_{M}\right)$ for all $M$ then $K_{0}(A) \approx \mathbf{Z} \oplus \operatorname{Pic}(A)$.

(3) From (iv) of Theorem 5 and (2) above one easily deduces: If $R$ is normal and semilocal with $2 R=R$, then $\nu(M)=\nu\left(M A_{M}\right)$ for all $M$ if and only if, all $A$-projectives are free.

(4) We know of no example of a normal semilocal $R$ for which $A$ has a nonfree projective; but we do know of examples of seminormal local $R$ for which $2 R=R$ and $K_{0}(A) \neq \mathbf{Z}(\operatorname{Pic}(A)=0$ for such $R)$.

ADDED IN PROOF. Case (iii) of Theorem 5 is proved with no restriction on Serre-dim $(R)$ in a forthcoming note, Generators for certain ideals in regular polynomial rings of dimension three, by the authors and M. Boratyniski.

5. Remarks on the nonnoetherian case. We now abandon the noetherian restriction on $R$, but retain in force the rest of the notation adopted in $\S 1$. It is known that $\nu(M)$ will be finite provided that $\nu(P)$ is. (In fact $\nu(M) \leqslant \nu\left(P R_{p}\right.$ ) $+n+1$ [D-G].) Recall the remark immediately following the proof of Theorem 2: all the points comprising that proof remain valid even if $R$ is not noetherian provided only that $\nu(P)$ is finite. Hence:

TheOREM $2^{\prime} \cdot \nu(M)=\nu\left(M A_{M}\right)$ if $P$ is maximal and $\nu(P)$ is finite. (Hence $\nu(M)=\nu\left(M A_{M}\right)$ whenever $R$ is a Hilbert ring, provided that $\nu(P)$ is finite. $)$

The statement of Theorem 3 also makes perfectly good sense for arbitrary $R$. Recall that its proof consisted of a preparation for the use of Theorem 2 via an application of Lemma 2. We have just seen that Theorem 2 remains valid in general; hence if the same were true of Lemma 2 so it would be with Theorem 3. We do not know whether this is the case; but an examination of our proof reveals that, in effect, we employ the noetherian property of $R$ only to establish two points: (1) $R / P$ is semilocal and $\operatorname{dim}(R / P) \leqslant 1$; (2) for all $n$ the polynomial $(R / P)$-algebra $A / P A$ has dimension $\operatorname{dim}(R / P)+n$. Prop- 
erty (1) may fail in general, but not if $R$ itself is semilocal of dimension 1 . Since Seidenberg initiated the general study of property (2), we say that a ring $S$ has the Seidenberg property provided that, for every $M$, the polynomial ring in $n$ variables over $S$ has dimension $\operatorname{dim}(S)+n$. Seidenberg [Sg] proves that Prüfer domains have this property and that a 1-dimensional domain has this property if, and only if, its normalization is a Prüfer domain. (Recall that a domain is a Prüfer domain if all of its localizations are valuation domains.) With these remarks at hand we assert that the proof of Lemma 2 was argued so as to be straightforwardly extendible to the case wherein $R$ is a polynomial ring in finitely many variables over a semilocal ring $S$, of dimension at most 1 , having the Seidenberg property. Therefore we have

TheOREM $3^{\prime}$. Assume $n>1$ and $\nu(P)$ finite. Then $\nu(M)=\nu\left(M A_{M}\right)$ provided that $R / P$ is semilocal, at most 1-dimensional and has the Seidenberg property.

Observe that the restrictions on $R / P$ are automatically satisfied if $R$ is noetherian or a 1-dimensional Prufer domain. Thus, modulo Lemma 3 below, we have as a corollary a result proved in [E] for $R$ a semilocal principal ideal domain. The proof of the lemma is an exercise in the use of Gauss' Lemma; we omit it.

Corollary. Assume $R$ is a 1-dimensional semilocal Priffer domain. Then: $\nu(M)=n$ if $P=0 ; \nu(M)=n+1$ if $P \neq 0$ and $\nu(P)$ is finite.

Lemma 3. Assume: $n=1 ; R$ is a normal domain; $P=0$. Let $J$ denote the Jacobson radical of $R$. Then $M=f A$, where $f=1+g$ and $g \in J A$.

Lastly we have the promised generalization of Theorem 4 . As before, $J$ and $\bar{J}$ denote the Jacobson radicals of $R$ and its normalization, respectively.

THEOREM $4^{\prime}$. Assume that $n=1$ and that $R$ is a 1-dimensional domain. Then $\nu(M)=\nu\left(M A_{M}\right)$ whenever $\nu(P)$ is finite if, and only if, $J=\bar{J}$.

Proof (CF. [E, 3.1]). Theorem $2^{\prime}$ applies if $R$ is a Hilbert ring; and this is the case if, and only if, $J=0$ (equivalently, $\bar{J}=0$ ). So we assume that $J \neq 0$; and by Theorem $2^{\prime}$ we need only consider $M$ for which $P=0$. Suppose that $J=\bar{J}$. Let $\bar{M}$ be a maximal ideal of the normalization $\bar{A}$ of $A$ lying over $M$. By Lemma $4 \bar{M}=f \bar{A}$, where $f=1+g$ and $g \in J \bar{A}=J A$; i.e., $f \in M$. For $h \in M, h=f u, u \in \bar{A} . h=u+u g$; whence $u \in A$ since $u g \in J \bar{A}=J A$. Hence $M=f A$; i.e., $\nu(M)=\nu\left(M A_{M}\right)=1$. Now suppose that $J \neq \bar{J}$, and let $x \in \bar{J}-J$. Since $R[x]$ is a finite $R$-module, whence the conductor of $R[x]$ is 
nonzero, some power of $x$ lies in $J$. So we may assume that $x \notin R$ and $x^{2}=a \in J$. Say $A=R[X]$. Note that $X^{2}-a$ is irreducible in $A$ : if not, then $a=b^{2}$ for some $b \in R$, and it follows that $x= \pm b$. Then $f=a X^{2}-1$ must be irreducible too. Let $Q$ be any prime ideal of $A$ containing $f . Q \cap R=0$ since $a \notin Q$; whence every such $Q$ must be a maximal ideal. Let $M$ be one such maximal ideal. We know that $\nu\left(M A_{M}\right)=1$; suppose that $\nu(M)=1$. Then it must be the case that $M=f A$. It follows that $f$ generates the extension of $M$ to the polynomial ring over the quotient field of $R$. This is impossible since $f$ factors over this field: $f=(x X+1)(x X-1)$.

\section{REFERENCES}

[A-T] E. Artin and J. T. Tate, A note on finite ring extensions, J. Math. Soc. Japan 3 (1951), 74-77. MR 13, 427.

[B] H. Bass, Libération des modules projectifs sur certain anneaux de polynômes, Séminaire Bourbaki 1973/1974, Exposé no. 448, Lecture Notes in Math., vol. 431, Springer-Verlag, Berlin and New York, 1975, pp. 228-254. MR 51 \# 18.

[B-M] H. Bass and M. P. Murthy, Grothendieck groups and Picard groups of abelian group rings, Ann. of Math. (2) 86 (1967), 16-73. MR 36 \#2671.

[Bo] E. Bombieri, Seminormalità e singolarità ordinarie, Symposia Mathematica, Vol. XI (INDAM, Rome, 1971), Academic Press, New York and London, 1973, pp. 205-210. MR 49 \#11361.

[D-1] E. D. Davis, Ideals of the principal class, $R$-sequences and a certain monoidal transformation, Pacific J. Math. 20 (1967), 197-205. MR 34 \#5860.

[D-2] ___ Regular sequences and minimal bases, Pacific J. Math. 26 (1971), 323-326. MR 43 \#1968.

[D-3] _ On the geometric interpretation of seminormality (in preparation).

[D-G] E. D. Davis and A. V. Geramita, Maximal ideals in polynomial rings, Conf. on Commutative Algebra, Lecture Notes in Math., vol. 311, Springer-Verlag, Berlin and New York, 1973, pp. 57-60. MR 49 \#2697.

[E] S. Endô, Projective modules over polynomial rings, J. Math. Soc. Japan 15 (1963), 339-352. MR 27 \#5808.

[Fd] D. Ferrand, Courbes gauches et fibres de rang 2, C. R. Acad. Sci. Paris Ser. A 281 (1975), A345-A347.

[F] O. Forster, Über die Anzahl der Erzeugenden eines Ideals in einem Noetherschen Ring, Math. Z. 84 (1964), 80-87; erratum, ibid. 86 (1964), 190. MR 29 \# 1231.

[E-E] D. Eisenbud and G. Evans, Three conjectures about modules over polynomial rings, Conf. on Commutative Algebra, Lecture Notes in Math., vol. 311, Springer-Verlag, Berlin and New York, 1973, pp. 78-89. MR 48 \#8466.

[G] A. V. Geramita, Maximal ideals in polynomial rings, Proc. Amer. Math. Soc. 41 (1973), 34-36. MR 47 \#6672.

[Mo] T. T. Moh, On the unboundedness of generators of prime ideals in power series rings of three variables, J. Math. Soc. Japan 26 (1974), 722-734. MR 50 \#7158.

[M] M. P. Murthy, Projective A[X]-modules, J. London Math. Soc. 41 (1966), 453-456. MR 34 \#188.

[N] M. Nagata, Local rings, Interscience, New York, 1962. MR 27 \#5790.

[Q] D. Quillen, Projective modules over polynomial rings, Invent. Math. 36 (1976), 167-171.

[S] P. Salmon, Singolarità e gruppo di Picard, Symposia Mathematica, Vol. II, (INDAM, Rome, 1968), Academic Press, New York and London, 1969, pp. 341-345. MR 40 \# 4263.

[Sg] A. Seidenberg, On the dimension theory of rings. II, Pacific J. Math. 4 (1954), 603-614. MR 16,441 . 
[Sr] J.-P. Serre, Sur les modules projectif, Séminaire Dubreil-Pisot (1960/1961), No. 2, Sécretariat Mathématique, Paris, 1963. MR 28 \#3911.

[Sw] R. Swan, Serre's problem, Conf. on Commutative Algebra-1975 (A. V. Geramita, Editor), Queen's Papers in Pure and Applied Math., no. 42, Queen's Univ., Kingston, Ontario, Canada.

[T] C. Traverso, Seminormality and Picard group, Ann. Scuola Norm. Sup. Pisa (3) 24 (1970), 585-595. MR 43 \#3275.

Department of Mathematics, State University of New York, Albany, New York 12222

Department of Mathematics, Queen's University, Kinston, Ontario, Canada 